\title{
Variant Designs and Characteristics of Improved Microstructured Solid-State Neutron Detectors
}

\author{
S.L. Bellinger, Member, IEEE, W.J. McNeil, D.S. McGregor, Member, IEEE
}

\begin{abstract}
Perforated semiconductor neutron detectors are compact, high-efficiency, diode detectors that operate at low power. Microstructured neutron detector fabrication methods have been improved over previous manufacturing methods. The neutron detectors are easily fabricated from high purity $n$-type $\mathrm{Si}$, in which patterned trenches are etched into the Si substrate, wherein shallow p-type junctions are diffused. The trenches are then backfilled with ${ }^{6} \mathrm{LiF}$ powder, making the devices sensitive to reaction products from the ${ }^{6} \mathrm{Li}(\mathrm{n}, \mathrm{t})^{3} \mathrm{He}$ reaction. Pulse height spectra show improved signal-to-noise ratio, higher neutron counting efficiency, and excellent gamma-ray discrimination over previous microstructured neutron detector designs. Thermal neutron detection measurements from a $0.0253 \mathrm{eV}$ diffracted neutron beam, yielded $20.4 \%$ intrinsic detection efficiency for devices with 245 micron deep trenches and $21 \%$ intrinsic detection efficiency for two back-to-back devices each having 113 micron deep trenches.
\end{abstract}

\section{INTRODUCTION}

$\mathrm{M}$ icrostructured semiconductor neutron detectors have been developed and studied at Kansas State University for many years [1-15]. Similar work is also under investigation by other groups [16-17]. The devices are constructed by etching features into a semiconductor substrate, diffusing a $p n$ junction within the perforations, and subsequently backfilling the trenches with neutron reactive material. The device structure has the potential of achieving intrinsic thermal neutron detection efficiencies, $\varepsilon_{\mathrm{tn}}$, greater than $35 \%[4,7,11$, 15]. Common ${ }^{6} \mathrm{LiF}$ and ${ }^{10} \mathrm{~B}$ thin-film coated diode detectors are inherently restricted to $\varepsilon_{\mathrm{tn}}$ values no greater than $5 \%$, with moderate low-channel gamma-ray sensitivity [18]. In addition, with proper alignment, two microstructured devices may be combined to compose a single detector with double the efficiency as a single microstructured device.

In the present work, the backfilled neutron reactive material is ${ }^{6} \mathrm{LiF}$, which relies on the ${ }^{6} \mathrm{Li}(\mathrm{n}, \mathrm{t})^{4} \mathrm{He}$ reaction. When thermal neutrons are absorbed by ${ }^{6} \mathrm{Li}$, a $2.73 \mathrm{MeV}$ triton and a 2.05 $\mathrm{MeV}$ alpha particle are ejected in opposite directions. These reaction products are much more energetic than those of the ${ }^{10} \mathrm{~B}(\mathrm{n}, \alpha){ }^{7} \mathrm{Li}$ or ${ }^{157} \mathrm{Gd}(\mathrm{n}, \gamma){ }^{158} \mathrm{Gd}$ reactions, such that the measured signal to noise ratio is larger, thereby improving discrimination of background radiations. ${ }^{6} \mathrm{Li}$ has a relatively large microscopic thermal neutron absorption cross section of

Manuscript received November 13, 2009. This work is supported in part by the Defense Threat Reduction Agency, contract DTRA-01-03-C-0051, National Science Foundation, Grant DE-FG07-04ID14599, and the US Department of Energy, NEER Program grant No. DE-FG07-04ID14599.

S.L. Bellinger, (telephone: 785-532-7087, e-mail: slb3888@ksu.edu), W.J. McNeil, D.S. McGregor are with the SMART Laboratory of Kansas State Univ., Manhattan, KS USA 66506.
940 b. Additionally, the stable compound ${ }^{6} \mathrm{LiF}$ was used instead of the highly reactive lithium metal.

The use of inductively-coupled-plasma reactive-ion-etching (ICP-RIE) high-aspect ratio deep etching (HARDE) techniques were used to etch unique microstructures in the $\mathrm{Si}$ based neutron detectors [5-7, 9, 10]. The Si microstructured diodes are designed by incorporating dopants inside the perforations as well as upon the top surface, thereby making a $p n$ junction within the perforations [3]. The diffusion process consumes surface damage and contamination caused by the reactive ion etching process, thereby reducing the reverse bias leakage current of the diode. As a result, pulse height spectra are more distinct, move further from the noise floor and exhibit the expected shapes predicted elsewhere $[11,15]$.

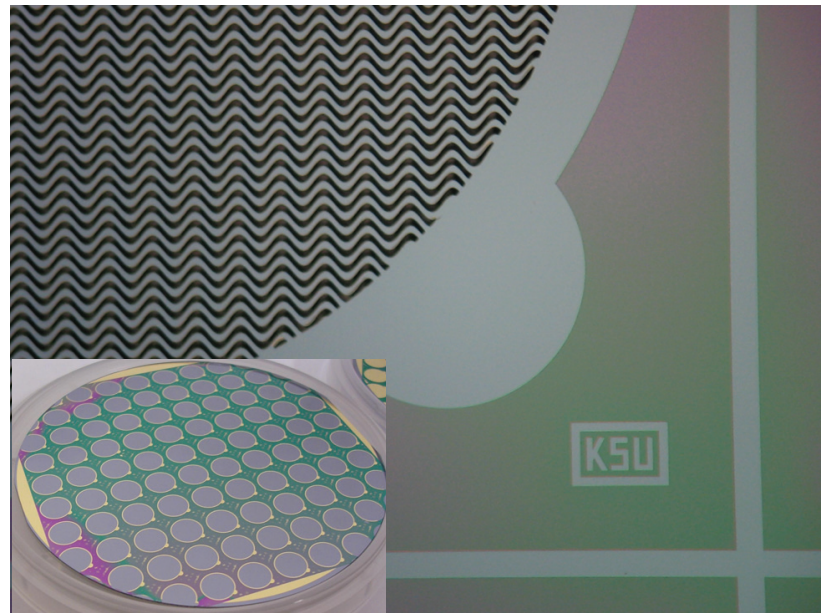

Fig. 1. Angular response of the device due to streaming effects is reduced by using sinusoidal perforation patterns. The pattern increases the intrinsic thermal neutron detection efficiency while producing a flat detection response. The inset shows an array of individual devices patterned on a 3 inch wafer.

\section{NEUTRON DETECTOR FABRICATION}

Initially, an oxide is grown on a $10 \mathrm{k} \Omega$-cm $n$-type $\mathrm{Si}$ wafer in which a diffusion window is patterned. Microstructured perforations are then etched into the $\mathrm{Si}$ diffusion windows with an ICP-RIE process. Individual detectors are batch processed on 3 inch diameter wafers, each with an active area of $0.28 \mathrm{~cm}^{2}$. The devices reported in the present work have sinusoidal etched trenches ranging between $22-26$ microns wide by 113 microns deep and 245 microns deep. The sinusoidal design maintains high neutron detection efficiency while generating a uniform angular response throughout a wide solid-angle around the normal incidence [5, 7-9]. After the etch process, the wafer is chemically cleaned and $p$-type regions are diffused uniformly into individual device microstructures across the wafer, thereby forming $p n$ junctions 


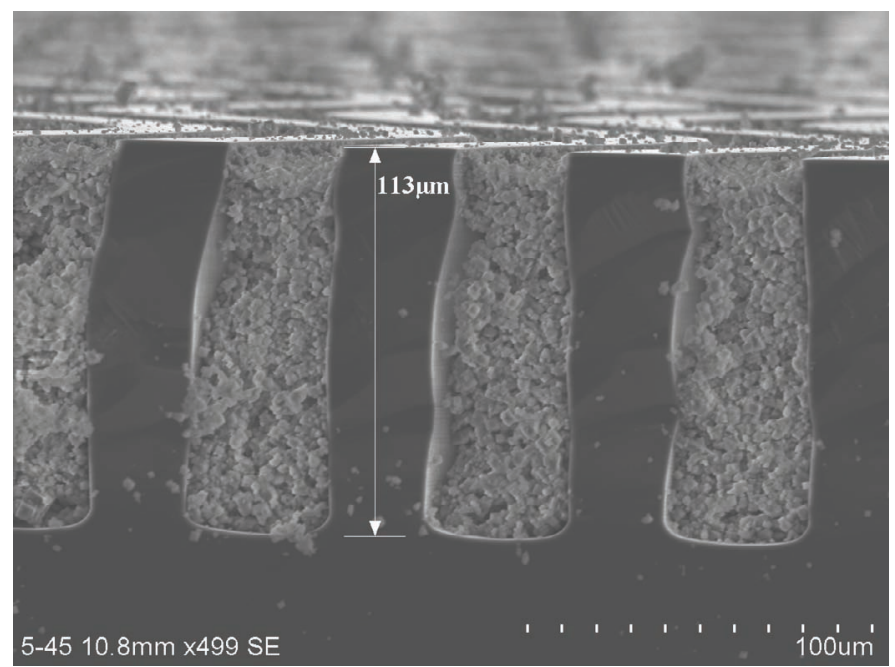

Fig. 2. ${ }^{6} \mathrm{LiF}$ powder is backfilled into the sinusoidal 113 micron deep perforations.

within the trenches. A Ti-Au metal contact is fabricated on the backside of the wafer to make an electrical ground contact, thereby completing the diode structure and enabling depletion through the bulk of the individual devices. Finally, ${ }^{6} \mathrm{LiF}$ powder is packed into the perforations to function as the neutron absorbing converter material (Fig. 2 \& Fig. 3). The devices are then mounted in emf shielded canisters for testing. More detail is discussed elsewhere [14].

\section{NeUtron Detection Measurements}

Neutron counting efficiency was measured with a 0.0253 $\mathrm{eV}$ diffracted neutron beam from the Kansas State University TRIGA Mark II nuclear reactor. The neutron flux was calibrated with a Reuter-Stokes ${ }^{3} \mathrm{He}$ gas-filled proportional detector and found to be $1.05 \pm 0.02 \times 10^{4} \mathrm{n} \mathrm{cm}^{-2} \mathrm{~s}^{-1}$. Details of the calibration method can be found elsewhere [13]. Pulse height spectra were collected from a sample perforated detector in the diffracted neutron beam with and without a $\mathrm{Cd}$ shutter, thereby allowing the collection of responses with and without thermal neutrons. Prompt gamma-rays emitted from the thin Cd shutter appear in the spectrum as numerous pulses at low energy near the noise floor of the detector system (Figs. 4,5 and 6 ). The neutron counting efficiency was calculated by dividing the neutron counts, collected from the detector with a lower level discriminator (LLD) set above the system noise, by the flux determined with the ${ }^{3} \mathrm{He}$ detector.

Fig. 4 shows pulse height spectra from a sinusoidal device with 113 micron deep etched features. The device works well with as little as 1 volt of applied bias. With the $\mathrm{Cd}$ shutter closed, the gamma-ray component (enhanced by the $\mathrm{Cd}$ shutter prompt gamma rays) was negligible at a LLD setting above channel 10 . The efficiency $\varepsilon_{\text {tn }}$ was determined by first subtracting the background gamma-ray component from the total neutron spectrum and then dividing by the measured neutron fluence. With the LLD set to channel $10, \varepsilon_{\mathrm{tn}}$ was measured to be $12.7 \%$.

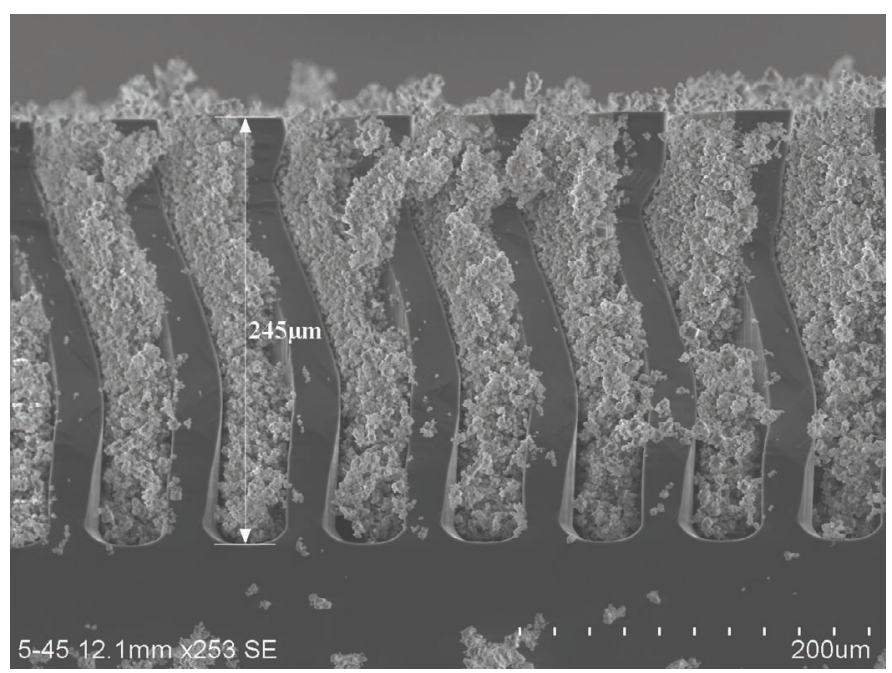

Fig. 3. ${ }^{6} \mathrm{LiF}$ powder is backfilled into the sinusoidal 245 micron deep perforations.

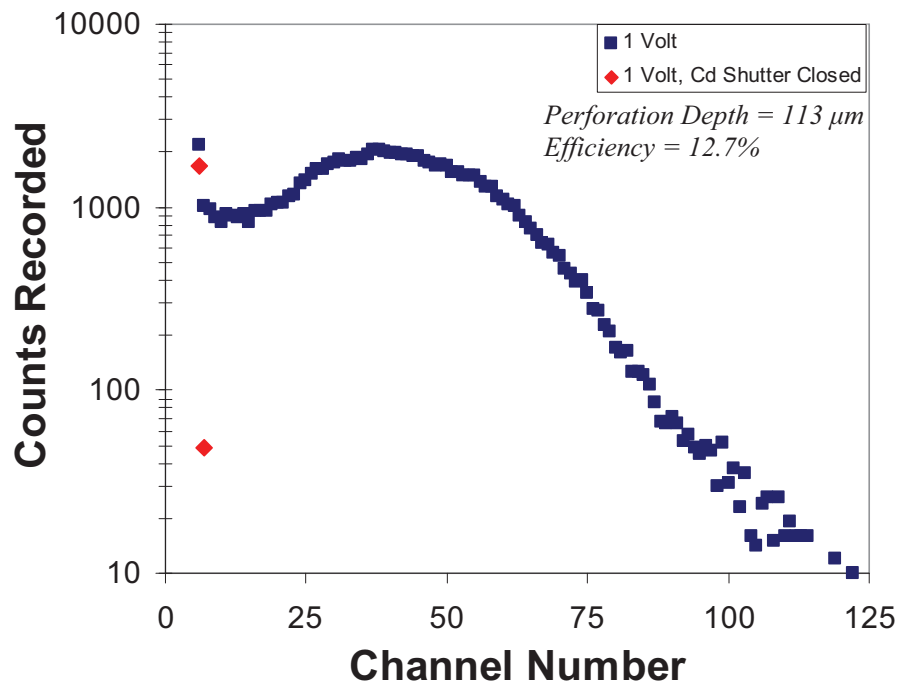

Fig. 4. Pulse height spectra for $113 \mu \mathrm{m}$ deep sinusoidal patterns backfilled with ${ }^{6} \mathrm{LiF}$. At $\mathrm{LLD}=10 \mathrm{Chn}$, the measured thermal neutron detection $\varepsilon_{\mathrm{tn}}$ was $12.7 \%$.

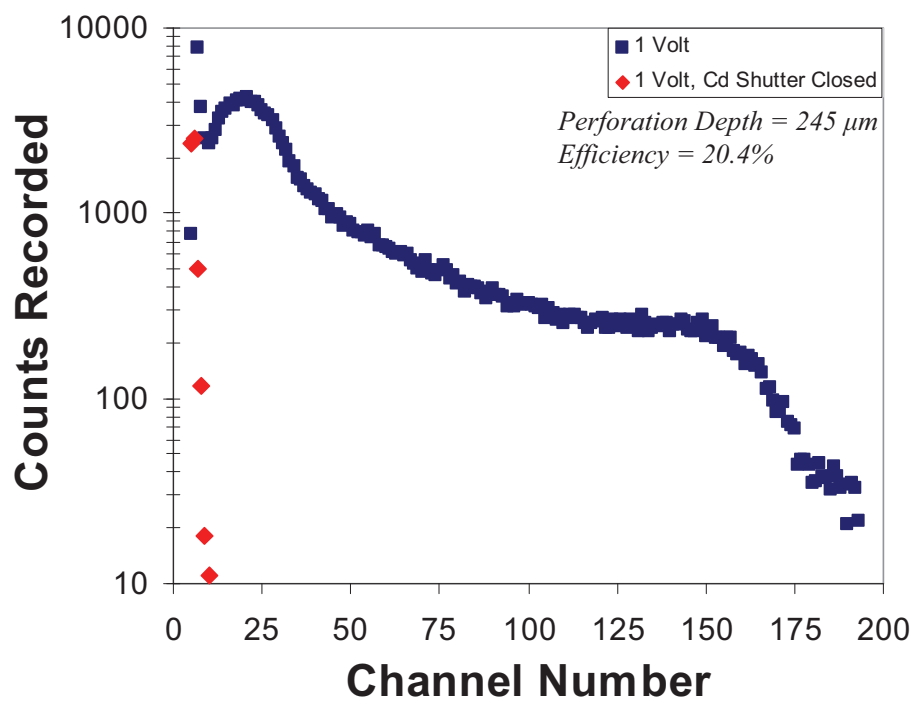

Fig. 5. Pulse height spectra for $245 \mu \mathrm{m}$ deep sinusoidal patterns backfilled with ${ }^{6} \mathrm{LiF}$. At $\mathrm{LLD}=10 \mathrm{Chn}$, the measured thermal neutron detection $\varepsilon_{\mathrm{tn}}$ was $20.4 \%$. 


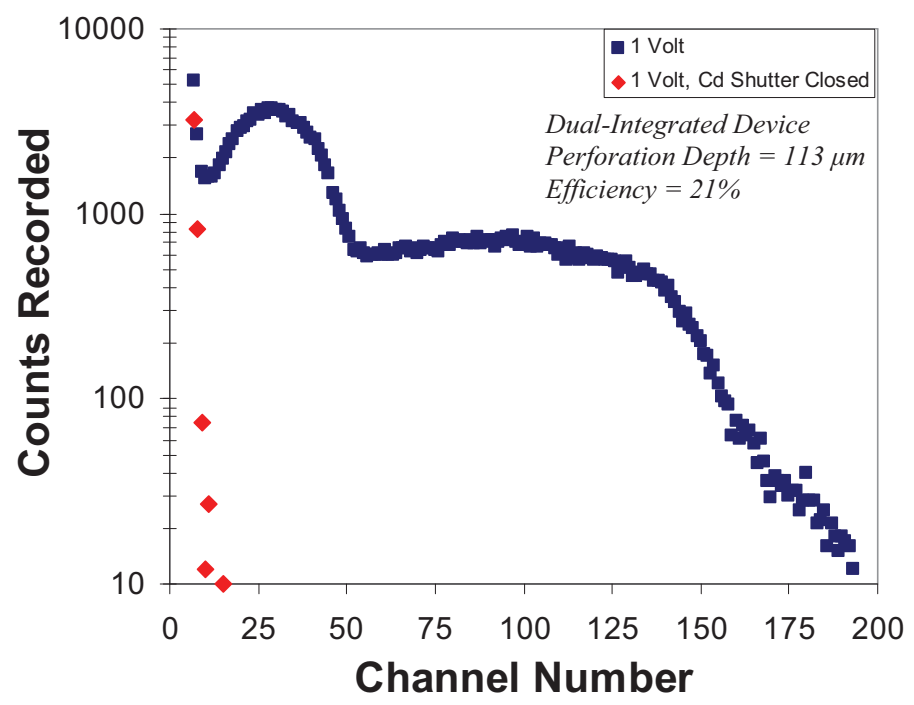

Fig. 6. Pulse height spectra for dual-integrated $113 \mu \mathrm{m}$ deep sinusoidal patterns backfilled with ${ }^{6} \mathrm{LiF}$. At LLD $=15 \mathrm{Chn}$, the measured thermal neutron detection $\varepsilon_{\mathrm{tn}}$ was $21 \%$.

Fig. 5 shows pulse height spectra from a sinusoidal device with 245 micron deep etched features. The device works well with as little as 1 volt of applied bias. Note that, compared to the 113 micron device, there is a downward shift of the pulse height signal from the detector. This downward shift is likely due to an increase in the capacitance of the device from the greater depth of the perforations. With the Cd shutter closed, the gamma-ray component was negligible at an LLD setting above channel 10 . The efficiency $\varepsilon_{\text {tn }}$ was determined by first subtracting the background gamma-ray component from the total neutron spectrum and then dividing by the measured neutron fluence. With the LLD set to channel $10, \varepsilon_{\mathrm{tn}}$ was measured to be $20.4 \%$.

Fig. 6 shows pulse height spectra from two back-to-back devices, each with 113 micron deep trenches, sandwiched together into a dual-integrated sinusoidal device. The single dual-integrated device works well with as little as 1 volt of applied bias. The pulse height spectrum shows many more counts than a single 113 micron deep etched device. Notice that the pulse height spectrum is slightly different from a single 113 micron device and appears more like the 245 micron device. The spectrum shows a downward shift of the pulse height signal from the detector. Though the spectrum of a dual-integrated device has not yet been modeled, this shift may again be due to an overall increase in capacitance of the stacked device, thereby reducing the pulse height signal from the detector. Similar to the other tested devices with the $\mathrm{Cd}$ shutter closed, the gamma-ray component was negligible at a LLD setting above channel 15. The efficiency $\varepsilon_{\text {tn }}$ was determined by first subtracting the background gamma-ray component from the total neutron spectrum and then dividing by the measured neutron fluence. With the LLD set to channel $15, \varepsilon_{\mathrm{tn}}$ was measured to be $21 \%$.

The actual gamma-ray flux with the Cd shutter in place was not measured; however, the $\mathrm{n} / \gamma$ ratio for the detector was determined using a ${ }^{60} \mathrm{Co}$ source directly on top of the device, to be 1.8 times larger than a $5 \mathrm{~cm}$ diameter, $10 \mathrm{~cm}$ long ${ }^{3} \mathrm{He}$ detector operated under identical measurement conditions [13]. Finally, the spectral shapes of the spectra in Figs. 4, 5 and 6 correspond well with the expected results calculated elsewhere, in which a dip in the spectrum appear in the region between $100 \mathrm{keV}$ and $800 \mathrm{keV}[11,15]$. Notice that this dip in counts at low energies allows for the trench design to be operated with an increased LLD setting over other microstructured designs, thereby improving gamma-ray discrimination, without severely reducing the detector efficiency. Deeper microstructured trenches and dualintegrated devices show a slight reduction in pulse height, which can decrease the $n / \gamma$ ratio. Yet, in the present work, this pulse height shift does not appear to be severe. Note that the gamma-ray interactions within the device also diminish with deeper perforations (due to less interaction volume), thereby allowing for smaller LLD settings resulting in superior gamma-ray discrimination.

\section{CONCLUSIONS}

New variant designs of the perforated $\mathrm{Si}$ detectors with sinusoidal trenches backfilled with ${ }^{6} \mathrm{LiF}$ powder have been characterized for neutron sensitivity in a diffracted $0.0253 \mathrm{eV}$ thermal neutron beam from a TRIGA Mark II nuclear reactor. An important clear advantage for the perforated microstructured neutron detectors is the high efficiency achieved with a single device. Furthermore, these devices can be dualintegrated into a single device to dramatically increase the counting efficiency of the neutron detector. Detectors with 113 micron deep trenches achieved $12.7 \% \varepsilon_{\mathrm{tn}}$, detectors with 245 micron deep trenches achieved $20.4 \% \varepsilon_{\text {tn }}$, and dualintegrated detectors with two 113 micron deep trenched devices achieved $21 \% \varepsilon_{\mathrm{tn}}$. The improved fabrication design with $p n$ junctions diffused within the perforations has lowered the leakage current and improved the pulse height signal from the devices. Spectra from the detectors match well to predicted results shown elsewhere $[11,15]$.

\section{REFERENCES}

[1] D.S. McGregor, R.T. Klann, H.K. Gersch, E. Ariesanti, J.D. Sanders, and B. Van Der Elzen, "New Surface Morphology for Low Stress ThinFilm-Coated Thermal-Neutron Detectors," IEEE Trans. Nucl. Sci., 49, pp. 1999-2004, 2002.

[2] D.S. McGregor and R.T. Klann, "Pocked Surface Neutron Detector," patent US-6545281; allowed April 8, 2003.

[3] D.S. McGregor, R.T. Klann, "High-Efficiency Neutron Detectors and Methods of Making the Same," patent US-7164138; allowed January 16, 2007.

[4] J.K. Shultis and D.S. McGregor, "Efficiencies of Coated and Perforated Semiconductor Neutron Detectors," IEEE Trans. Nucl. Sci., NS-53, pp. 1659-1665, 2006.

[5] W.J. McNeil, S. L. Bellinger, T. C. Unruh, E. L. Patterson, J. K. Shultis, and D. S. McGregor, "Development of Perforated Si Diodes for Neutron Detection," IEEE Nucl. Sci. Symp. Conf. Rec., San Diego, CA, Oct. 29Nov. 3, 2006.

[6] W.J. McNeil, S.L. Bellinger, B.J. Blalock, C.L. Britton Jr., J.L. Britton, S.C. Bunch, W.L. Dunn, C.M. Henderson, T.J. Sobering, R.D. Taylor, T.C. Unruh, D. S. McGregor, "Preliminary Tests of a High Efficiency 1D Silicon Pixel Array for Small Angle Neutron Scattering," IEEE Nucl. Sci. Symp. Conf. Rec, Waikiki, Hawaii, Oct. 28-Nov. 3, 2007.

[7] S.L. Bellinger, W.J. McNeil, T.C. Unruh, D.S. McGregor, "Angular Response of Perforated Silicon Diode High Efficiency Neutron 
Detectors," IEEE Nucl. Sci. Symp. Conf. Rec, Waikiki, Hawaii, Oct. 28Nov. 3, 2007.

[8] C.J. Solomon, J.K. Shultis, W.J. McNeil, T.C. Unruh, B.B. Rice, and D.S. McGregor, "A Hybrid Method for Coupled Neutron-Ion Transport Calculations for ${ }^{10} \mathrm{~B}$ and ${ }^{6} \mathrm{LiF}$ Coated and Perforated Detector Efficiencies," Nucl. Instrum. and Meth., A580, pp. 326-330, 2007.

[9] D.S. McGregor, S.L. Bellinger, D. Bruno, W.J. McNeil, E. Patterson, J.K. Shultis, C.J. Solomon, T. Unruh, "Perforated Semiconductor Neutron Detectors for Battery Operated Portable Modules," Proc. SPIE, 6706, pp. 0N1-0N12, 2007.

[10] D.S. McGregor S. Bellinger, D. Bruno, W.J. McNeil, E. Patterson, B.B. Rice, "Perforated Semiconductor Neutron Detector Modules," Proc. of 32nd Annual GOMACTech Conf., Lake Buena Vista, FL, March 19-22, 2007.

[11] D.S. McGregor and J.K. Shultis, "Designs for Micro-Structured Semiconductor Neutron Detectors," Proc. SPIE, 7079, pp. 0601 - 0615, 2008.

[12] D.S. McGregor, S.L. Bellinger, W.J. McNeil, T.C. Unruh, "MicroStructured High-Efficiency Semiconductor Neutron Detectors," IEEE Nucl. Sci. Symp. Conf. Rec, Dresden, Germany, Oct. 19-Oct. 25, 2008.

[13] D.S. McGregor, W. J. McNeil, S. L. Bellinger, T. C. Unruh, J. K. Shultis, "Microstructured Semiconductor Neutron Detectors," Nucl. Instrum. and Meth., A608, pp. 125-131, 2009.

[14] S.L. Bellinger, W.J. McNeil, D.S. McGregor, "Improved Fabrication Technique for Microstructured Solid-State Neutron Detectors," Proc. MRS, vol. 1164, 2009.

[15] J.K. Shultis and D.S. McGregor, "Design and Performance Considerations for Perforated Semiconductor Thermal-Neutron Detectors," Nucl. Instrum. and Meth., A606, pp. 608-636, 2009.

[16] J. Uher, F. Frojdh, J. Jakubek, C. Kenney, Z. Kohout, V. Linhart, S. Parker, S. Petersson, S. Pospisil and G. Thungstrom, Characterization of 3D Thermal Neutron Semiconductor Detectors," Nucl. Instrum. and Meth., A576, pp. 32 - 37, 2007.

[17] R.J. Nikolic, A.M. Conway, C.E. Reinhardt, R.T. Graff, T.F. Wang, N. Deo and C.L. Cheung, "6:1 Aspect Ratio Pillar Based Thermal Neutron Detector Filled with ${ }^{10} \mathrm{~B}$," Appl. Phys. Lett., 93, pp. 133502.1 133502.3, 2008.

[18] D.S. McGregor, M.D. Hammig, H.K. Gersch, Y-H. Yang, and R.T. Klann, "Design Considerations for Thin Film Coated Semiconductor Thermal Neutron Detectors, Part I: Basics Regarding Alpha Particle Emitting Neutron Reactive Films," Nucl. Instrum. and Meth., A500, pp. 272-308, 2003. 